\title{
Pelvic Organ Prolapse during Pregnancy: A Case Series and Review of Literature
}

Chanderdeep Sharma*, Manupriya Sharma, Anjali Soni, Pawan Kumar Soni, Ashok Verma and Suresh Verma

DR R P G M C Kangra, Tanda (HP), India

\begin{abstract}
Pelvic organ prolapse during pregnancy is extremely rare. Limited cases (less than 30) have been reported since 1980. From a very benign presentation of heaviness in perineum, it can present as uterine rupture with fetal and maternal mortality. No standard guidelines of care have been established for this rare presentation. There is gross variation in management ranging from conservative measures, laparoscopic surgery to cesarean section followed by peri-partum hysterectomy and abdominal sacral colpopexy. This case series report five cases of pelvic organ prolapse during pregnancy and outlines an approach of watchful expectancy with favorable maternal \& fetal outcomes.
\end{abstract}

Keywords: Pelvic organ prolapsed; Pregnancy; Cervix

\section{Introduction}

Pelvic organ prolapse (POP) is a common gynecological complaint in developing countries (mean prevalence 19.7\% [range 3.4-56.4\%]) [1]. However, POP during pregnancy; is a relatively rare condition with estimated incidence of 1 per 10,000-15,000 deliveries [2]. Furthermore; this incidence is declining with 39 cases reported since 1980 (Table 1) [2-33].

Prolapse of pelvic organs (usually cervix) during pregnancy is different from pregnancy in a woman with pre-existing POP. Whereas POP before pregnancy usually resolves spontaneously by the end of second trimester, the prolapse during pregnancy usually starts developing in second or third trimester and may worsen progressively if unattended [2-34]. We hereby report five cases of POP during pregnancy.

\section{Case 1}

A 34-year-old pregnant women, (gravida 3, para 2), presented to outpatient department at 37 weeks of gestation, with the feeling of something descending into her vagina for three weeks. All her previous deliveries were home conducted vaginal deliveries with no difficulty. There was no significant complaint except for abnormal sensation of mass in the perineum since 32 weeks of gestation. On examination there was there was stage 2 POP [pelvic organ prolapse quantification system (POP-Q)] (Figure 1), with no ulceration, edema

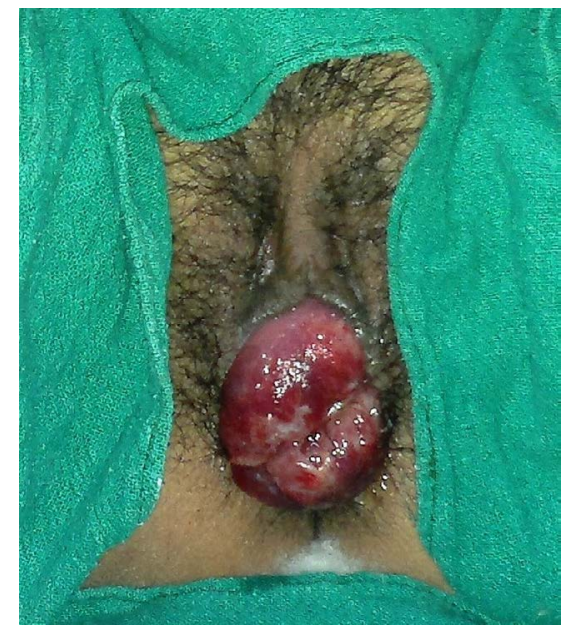

Figure 1: Pregnant women with POP [Q] stage 2 or desiccation of the cervix. She was hospitalized and advised bed rest in slight Trendelenburg's position. After bed rest alone her cervix was interiorized and descended usually during walking or straining. She had spontaneous labor onset at 37 weeks 5 days of gestation and had normal vaginal delivery. On subsequent follow up 3 months post partum, there was no residual prolapse.

\section{Case 2}

A 26-year-old pregnant women, (gravid 2, para1), presented with complaint of heaviness in perineum at 26 weeks of gestation. Her previous delivery was a normal vaginal delivery. On examination her cervix descended up to stage 2 POP-Q classification as shown in Figure 2. Cervix was healthy. She was advised bed rest in slight Trendelenburg's position. Her subsequent pregnancy was uneventful. She had spontaneous onset of labor at 39 weeks 5 days of gestation \& delivered a healthy baby. On subsequent follow up three months later there was no prolapse seen.

\section{Case 3}

A 32-year-old pregnant woman, gravid 2, para 1, presented to

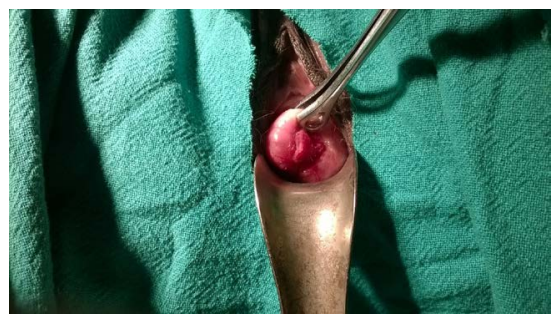

Figure 2: Pregnant women with POP [Q] stage 2 .

*Corresponding author: Chanderdeep Sharma, Assistant Professor (OBG), DR R P G M C Kangra, Tanda (HP), India-176001, Tel: 91-9218925471l; E-mail: cdsharma2006@gmail.com

Received October 14, 2014; Accepted October 31, 2014; Published November 15,2014

Citation: Sharma C, Sharma M, Soni A, Soni PK, Verma A, et al. (2014) Pelvic Organ Prolapse during Pregnancy: A Case Series and Review of Literature. Gen Med (Los Angel) 2: 151. doi: 10.4172/2327-5146.1000151

Copyright: $\odot 2014$ Sharma C, et al. This is an open-access article distributed under the terms of the Creative Commons Attribution License, which permits unrestricted use, distribution, and reproduction in any medium, provided the original author and source are credited. 